\title{
Prognostic impacts of the combined positive score and the tumor proportion score for programmed death ligand-1 expression by double immunohistochemical staining in patients with advanced gastric cancer
}

\author{
Kohei Yamashita ${ }^{1} \cdot$ Masaaki Iwatsuki $^{1,2} \cdot$ Kazuto Harada $^{1,2} \cdot$ Kojiro Eto $^{1} \cdot$ Yukiharu Hiyoshi $^{1} \cdot$ Takatsugu Ishimoto $^{1}$. \\ Yohei Nagai ${ }^{1}$. Shiro Iwagami ${ }^{1}$ - Yuji Miyamoto ${ }^{1}$. Naoya Yoshida ${ }^{1}$. Yoshihiro Komohara ${ }^{3}$. Jaffer A. Ajani ${ }^{2}$. \\ Hideo Baba' ${ }^{1}$ (B)
}

Received: 19 June 2019 / Accepted: 17 August 2019 / Published online: 26 August 2019

(c) The International Gastric Cancer Association and The Japanese Gastric Cancer Association 2019

\begin{abstract}
Background The tumor proportion score (TPS) and combined positive score (CPS) have been developed to assess programmed death ligand 1 (PD-L1) expression in gastric cancer (GC). This study aimed to elucidate the role of TPS and CPS as prognostic biomarkers.

Methods A total of 191 patients with GC who received curative gastrectomy were retrospectively enrolled. Double immunohistochemistry of PD-L1 and ionized calcium binding adaptor molecule 1 was performed to clearly distinguish PD-L1 expression between tumor cells and macrophages. Survival analysis for PD-L1 expression by TPS and CPS was performed. Results PD-L1 positivity was detected in 39 patients (20.4\%) by TPS and in 137 patients (71.7\%) by CPS. Patients with PD-L1 positivity by CPS experienced significantly shorter overall survival (OS) $(P<0.01)$ and relapse-free survival (RFS) $(P=0.01)$ than the other patients. In contrast, patients with either PD-L1 status by the TPS showed similar OS and RFS times. Multivariate Cox regression analysis for OS and RFS demonstrated that PD-L1 positivity by CPS was a significant independent factor for poor OS (hazard ratio [HR] 2.27, 95\% confidence interval [CI] 1.27-4.37, $P<0.01$ ) and RFS (HR $1.81,95 \%$ CI $1.07-3.22, P=0.03$ ).

Conclusions CPS was shown to be a more useful assessment method of determining PD-L1 expression than TPS as a prognostic biomarker. This suggests that the total number of PD-L1-expressing cells, including tumor and immune cells, is a more sensitive prognostic biomarker than the number of PD-L1-expressing tumor cells in GC.
\end{abstract}

Keywords Gastric cancer · PD-L1 · Combined positive score $\cdot$ Prognostic biomarker

Hideo Baba

hdobaba@kumamoto-u.ac.jp

1 Department of Gastroenterological Surgery, Graduate School of Medical Sciences, Kumamoto University, 1-1-1 Honjo, Chuo-ku, Kumamoto 860-8556, Japan

2 Department of Gastrointestinal Medical Oncology, The University of Texas MD Anderson Cancer Center, Houston, TX 77030, USA

3 Department of Cell Pathology, Graduate School of Medical Sciences, Kumamoto University, Kumamoto, Japan

\section{Introduction}

Gastric cancer (GC) is the fifth most common malignancy and the third leading cause of death from cancer worldwide [1]. Although surgical techniques and multidisciplinary treatments have been developed, long-term outcomes for patients with advanced GC remain poor. Therefore, there is an urgent needed to establish a novel prognostic biomarker to help select optimal treatment strategies for patients with advanced GC.

Recently, the programmed cell death 1 (PD-1)/programmed death ligand 1 (PD-L1) interaction has attracted attention as one of the major immune checkpoint pathways involved in inducing immune tolerance [2]. PD-1, which is 
mainly expressed on activated $\mathrm{T}$ cells, negatively regulates the immune response by binding its ligand, PD-L1. PD-L1 is typically expressed on various types of immune cells and some specific cancer cell types, and enables cancers to evade the immune system. Therefore, PD-L1 expression in tumors has been considered a potential prognostic biomarker in several types of cancer including melanoma and non-small cell lung carcinoma [3, 4]. However, inconsistent results on the prognostic impact of PD-L1 expression in GC have been reported $[5,6]$.

The most challenging problem researchers and clinicians facing is that a consensus of assessment criteria for PD-L1 status has not been established in GC. Recently, two scoring systems have been developed for the assessment of PD-L1 expression: the tumor proportion score (TPS) and the combined positive score (CPS). In TPS, PD-L1 expression in tumors is evaluated by the ratio of PD-L1-stained tumor cells to the total number of viable tumor cells. CPS calculates the ratio of potential PD-L1 expression including tumor cells and immune cells to the total number of viable tumor cells. These two scoring systems have been used in several important clinical trials on the therapeutic effect of PD-1 inhibitors in GC [7-9]. However, it remains unclear which is most suitable for the assessment of PD-L1 expression as a prognostic biomarker.

PD-L1 expression is usually evaluated with immunohistochemistry (IHC) against PD-L1 in clinical tissue samples. Therefore, it is necessary to distinguish tumor cells from other types of cells including immune cells to obtain an accurate assessment of PD-L1 expression by TPS. However, in clinical practice, it is difficult to histomorphologically distinguish poorly differentiated adenocarcinoma cells from macrophages, which both express PD-L1 on their cell surface. Consequently, no previous reports have considered the accurate assessment of PD-L1 expression by TPS and how this affects the prognostic importance. Moreover, it is also unclear whether TPS or CPS is a more useful prognostic biomarker for GC patients after accurate assessment of the PD-L1 status.

In this study, we performed double IHC of PD-L1 and the macrophage marker ionized calcium binding adaptor molecule 1 (Iba)-1 to distinguish between them and achieve an accurate assessment of PD-L1 expression by TPS. Based on this assessment, we performed survival analysis to elucidate and compare the prognostic significance of PD-L1 expression by TPS and CPS in patients with GC who received curative gastrectomy. In addition, we performed comprehensive survival analysis in combination with PD-L1 expression and tumor infiltrating lymphocytes (TILs).

\section{Materials and methods}

\section{Patients and samples}

This study included 191 patients with GC who received curative gastrectomy at the Department of Gastroenterological Surgery, Kumamoto University Hospital between February 2005 and January 2018. Advanced GC cases whose tumor depth was below the muscularis propria layer were eligible for this study. Patients who received pretreatment including neoadjuvant chemotherapy before curative surgery were excluded. Tumor staging was performed according to the American Joint Committee on Cancer Staging Manual 8 th edition [10]. Slides stained with hematoxylin and eosin (H\&E) were reviewed for each case and a representative block was chosen for IHC. Clinical information was collected from the patient's medical records. Written informed consent was obtained from all patients and this study procedure was approved by the Institutional Review Board of Kumamoto University Hospital (No. 1037).

\section{Double immunohistochemistry}

Formalin-fixed paraffin-embedded tissue samples were sectioned at $5 \mu \mathrm{m}$. After deparaffinization, heat-induced antigen retrieval was performed in a steamer autoclave at $121^{\circ} \mathrm{C}$ for $15 \mathrm{~min}$ with antigen retrieval solution $(\mathrm{pH} 9$, Histofine; Nichirei Biosciences, Tokyo, Japan). Endogenous peroxidase activity was blocked using $3 \%$ hydrogen peroxide. Slides were then incubated with a rabbit monoclonal primary antibody against PD-L1 (1:200 dilution; Cell Signaling Technology, Danvers, MA, USA) overnight at $4{ }^{\circ} \mathrm{C}$. Next, slides were incubated with anti-rabbit EnVision ${ }^{\mathrm{TM}}+/$ horseradish peroxidase secondary antibody (Dako Japan, Tokyo, Japan) for $1 \mathrm{~h}$. After visualization with 3, 3-diaminobenzidine, antigen retrieval was re-performed by microwaving for 5 min with antigen retrieval solution $(\mathrm{pH} \mathrm{7,} \mathrm{1:20} \mathrm{dilution;}$ LSI Medience Corporation, Tokyo, Japan).

After blocking with 3\% hydrogen peroxide, slides were incubated with a rabbit monoclonal primary antibody against Iba-1 (1:500 dilution; Wako; Osaka, Japan) for $1 \mathrm{~h}$. They were then incubated with an anti-rabbit EnVision + / horseradish peroxidase secondary antibody (Dako Japan) for $20 \mathrm{~min}$. Finally, slides were visualized with HistoGreen (HistoPrime, HRP-Substrate-Kit; Linaris, Dossenheim, Germany) and counterstained with hematoxylin.

\section{Evaluation of PD-L1 expression}

PD-L1 expression was evaluated using TPS and CPS. TPS was determined as the number of PD-L1-positive tumor cells 
divided by the total number of viable tumor cells, multiplied by 100 , which was substantially the same as a previously reported method [7]. CPS was calculated by summing the number of PD-L1-positive cells including tumor cells, lymphocytes, and macrophages, dividing this by the total number of viable tumor cells, and multiplying by 100, as used in a previous study [8]. Although the result of this calculation can exceed 100, the maximum score is defined as CPS 100. TPS and CPS were both calculated by two observers including an experienced pathologist (K.Y. and Y.K.) who were blind to clinical information. PD-L1 positivity was defined as the average score of the two observers if this was $\geq 1$ for both TPS and CPS. All samples were confirmed to include at least 100 viable tumor cells, which is regarded as adequate for PD-L1 assessment [11].

\section{Evaluation of TILs status}

TILs status was also evaluated using the matched hematoxylin and eosin (H\&E)-stained slides by the two observers. As there is no consensus on the evaluation for TILs status in GC, this study adopted and modified the criteria in previous studies $[12,13]$. In brief, the percentage of tumor nest and tumor stromal area that contains infiltrating lymphocytes were semi-quantitatively scored with four-point scale: score 0 (infiltrating lymphocyte is absent), score 1 (mild increase of infiltrating lymphocytes), score 2 (moderate increase of infiltrating lymphocytes) and score 3 (prominent increase of infiltrating lymphocytes). During subsequent scoring, any confusing cases were discussed by the two observers and determined the scores. We defined score $0-1$ as TILs low and score $2-3$ as TILs high.

\section{Statistical analysis}

Statistical comparisons between PD-L1-positive and -negative groups were performed using Wilcoxon tests for quantitative variables and Chi-square tests for qualitative variables. Overall survival (OS) was calculated as the time from surgery to death from any cause. If patients were alive at the last follow-up, they were censored. Relapse-free survival (RFS) was calculated as the time from surgery to recurrence or death from any cause. If patients were alive or recurrence-free at the last follow-up, they were censored. Survival curves for OS and RFS were described using the Kaplan-Meier method and log-rank tests were utilized for survival analysis. Prognostic values of PD-L1 positivity and other clinical factors were assessed using univariate and multivariate analyses based on the Cox proportional hazard regression model. Hazard ratios (HRs) and 95\% confidence intervals (CIs) were estimated for each factor. To assess interactions between variables, PD-L1 positivity was crosscorrelated with another variable of interest in the univariate logistic regression model, and the interaction was evaluated a

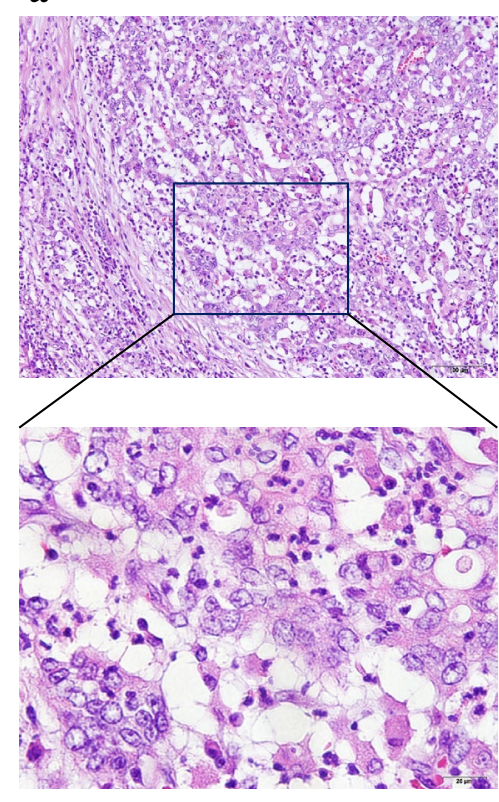

$\mathrm{H} \& \mathrm{E}$ b

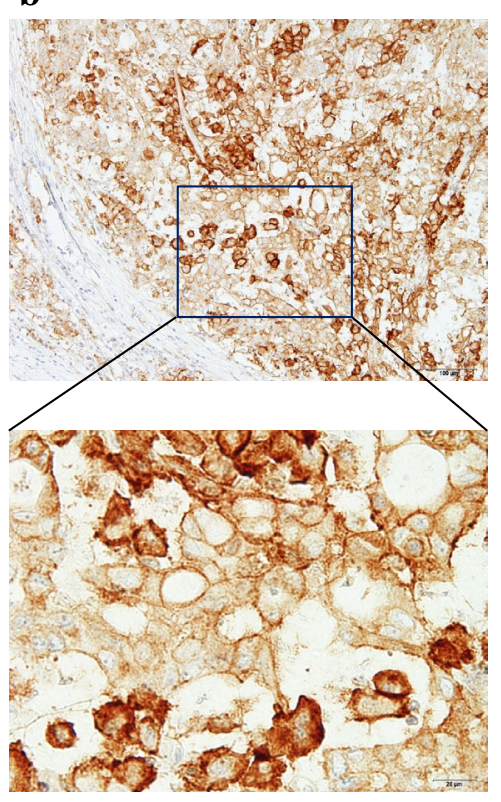

PD-L1 c

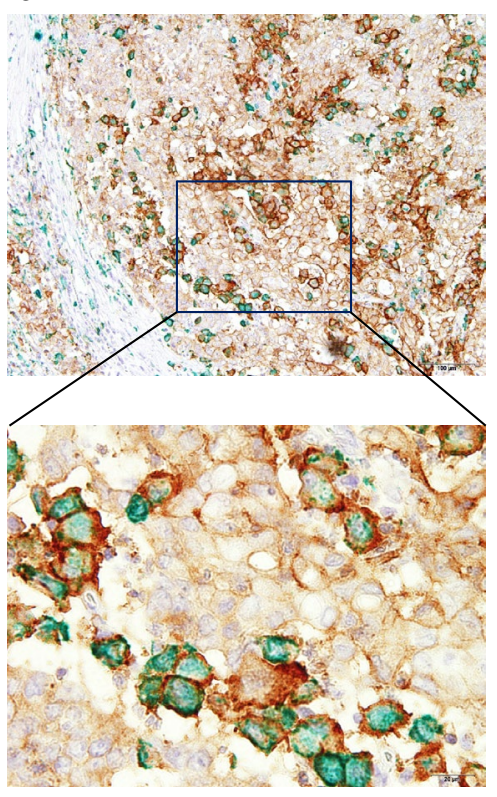

PD-L1 and Iba-1

Fig. 1 Representative images of double IHC for PD-L1 and Iba-1 in GC case. a H\&E staining. $\times 100$ (upper panel), $\times 400$ (lower panel). b IHC for PD-L1. $\times 100$ (upper panel), $\times 400$ (lower panel). c Double IHC for PD-L1 and Iba-1. $\times 100$ (upper panel), $\times 400$ (lower panel) 
by the likelihood ratio test. All statistical analyses were performed by JMP ${ }^{\circledR}$ version 13.1 software (SAS Institute, Cary, NC, USA). All $P$ values were two-sided and $P<0.05$ was considered statistically significant.

\section{Results}

\section{PD-L1 expression evaluated by TPS and CPS}

Figure 1 shows a representative double IHC for PD-L1 and Iba-1. PD-L1 expression was confirmed as convincing partial or complete linear membrane staining of a brown color whereas Iba-1 was confirmed as cytoplasmic staining of a green color. Although it was difficult to distinguish poorly differentiated adenocarcinoma cells and macrophages using H\&E staining and IHC samples stained for PD-L1 (Fig. 1a, b), we could distinguish them clearly using double IHC for PD-L1 and Iba-1 (Fig. 1c). For example, samples where PD-L1 is expressed in both tumor cells and macrophages are regarded as PD-L1-positive by TPS and CPS (Fig. 2a), while samples where PD-L1 is only expressed in macrophages are regarded as PD-L1-negative by TPS and PD-L1-positive by CPS (Fig. 2b).

According to the described methods, PD-L1 positivity was detected in 39 patients (20.4\%) by TPS and 137 patients (71.7\%) by CPS.

\section{Relationships between PD-L1 expression and clinicopathological factors}

The relationships between PD-L1 expression and clinicopathological factors are summarized in Table 1. Clinicopathological factors were similar between patients shown to be PD-L1-positive and those shown to be PD-L1-negative by TPS. In contrast, patients with PD-L1 positivity by CPS had a significantly higher elderly proportion (aged $\geq 65$ years; $P=0.03$ ) and a significantly higher differentiated tumor type $(P=0.04)$ than those with PD-L1 negativity.

b
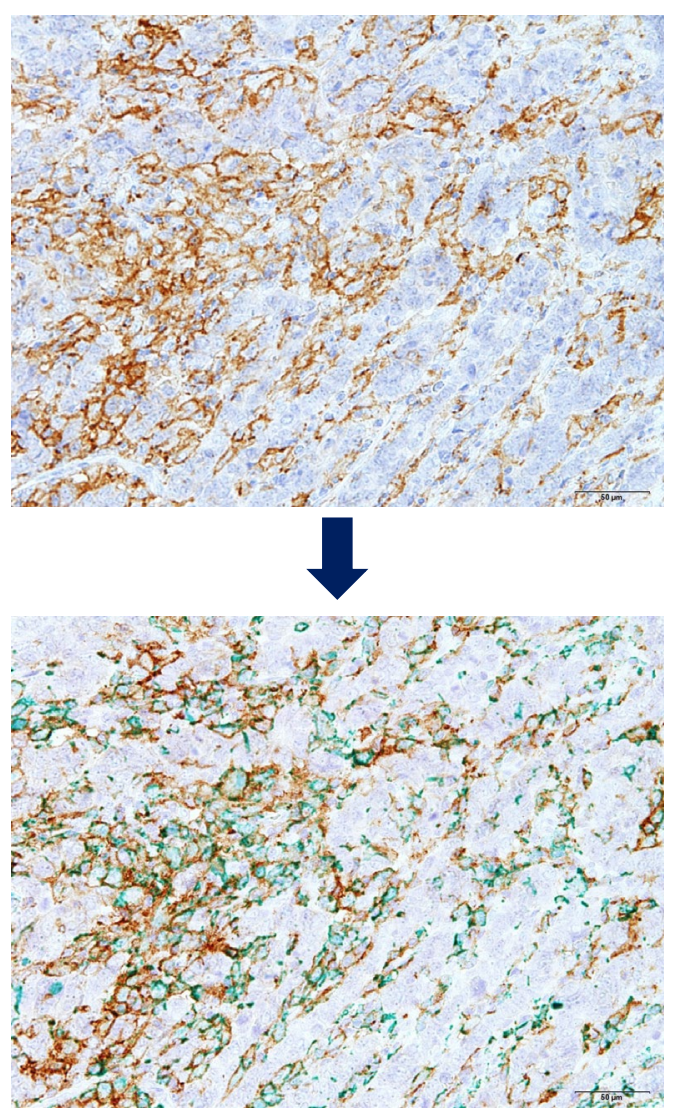

TPS: PD-L1 (-) CPS:PD-L1 (+)

Fig. 2 Examples of assessment of PD-L1. a Case with PD-L1-positivity by TPS and CPS. b Case with PD-L1-negativity by TPS and PDL1-positivity by CPS 
Table 1 Relationship between the PD-L1 expression and clinical parameter

\begin{tabular}{|c|c|c|c|c|c|c|}
\hline & \multicolumn{3}{|l|}{ TPS } & \multicolumn{3}{|l|}{ CPS } \\
\hline & Positive & Negative & $P$ value & Positive & Negative & $P$ value \\
\hline & $N=39(\%)$ & $N=152(\%)$ & & $N=137(\%)$ & $N=54(\%)$ & \\
\hline \multicolumn{7}{|l|}{ Age (years) } \\
\hline$<65$ & $8(20.5)$ & 44 (28.9) & 0.28 & $31(22.6)$ & $21(38.9)$ & 0.03 \\
\hline$\geq 65$ & $31(79.5)$ & $108(71.1)$ & & $106(77.4)$ & $33(61.1)$ & \\
\hline \multicolumn{7}{|l|}{ Sex } \\
\hline Male & $24(61.5)$ & $111(73.0)$ & 0.17 & $99(72.3)$ & $36(66.7)$ & 0.45 \\
\hline Female & $15(38.5)$ & $41(27.0)$ & & $38(27.7)$ & $18(33.3)$ & \\
\hline \multicolumn{7}{|l|}{ Tumor location } \\
\hline Upper & $12(30.8)$ & 45 (29.6) & 0.12 & $42(30.7)$ & $15(27.8)$ & 0.83 \\
\hline Middle & $9(23.1)$ & $59(38.8)$ & & $47(34.3)$ & $21(38.9)$ & \\
\hline Lower & $18(46.1)$ & 48 (31.6) & & $48(35.0)$ & $18(33.3)$ & \\
\hline \multicolumn{7}{|l|}{ Histological type } \\
\hline Differentiated & $20(51.3)$ & $77(50.7)$ & 0.94 & $76(55.5)$ & $21(38.9)$ & 0.04 \\
\hline Undifferentiated & $19(48.7)$ & $75(49.3)$ & & $61(44.5)$ & $33(61.1)$ & \\
\hline \multicolumn{7}{|l|}{ Tumor marker } \\
\hline $\mathrm{CEA}>3.4$ & $10(25.6)$ & 39 (25.6) & 0.99 & $38(27.7)$ & $11(20.4)$ & 0.28 \\
\hline CA19-9>37 & 7 (18.0) & $19(12.5)$ & 0.39 & $20(14.6)$ & $6(11.1)$ & 0.52 \\
\hline \multicolumn{7}{|l|}{ T stage } \\
\hline $\mathrm{T} 2$ & $13(33.3)$ & $37(24.3)$ & 0.26 & 35 (25.6) & 15 (27.8) & 0.75 \\
\hline T3-T4 & $26(66.7)$ & 115 (75.7) & & $102(75.4)$ & $39(72.2)$ & \\
\hline \multicolumn{7}{|l|}{$\mathrm{N}$ stage } \\
\hline No & $17(43.6)$ & $78(51.3)$ & 0.39 & $73(53.3)$ & $22(40.7)$ & 0.12 \\
\hline $\mathrm{N} 1-\mathrm{N} 3$ & $22(56.4)$ & 74 (48.7) & & $64(46.7)$ & $32(59.3)$ & \\
\hline \multicolumn{7}{|l|}{ Stage } \\
\hline I & $10(25.6)$ & $27(17.8)$ & 0.39 & $26(19.0)$ & $11(20.4)$ & 0.16 \\
\hline II & $18(46.2)$ & $67(44.1)$ & & $56(40.9)$ & $29(53.7)$ & \\
\hline III & $11(28.2)$ & $58(38.2)$ & & $55(40.1)$ & $14(25.9)$ & \\
\hline
\end{tabular}

Statistically significant $P$ values are in bold $(P<0.05)$

\section{PD-L1 expression and patient survival}

We next performed survival analysis to assess the prognostic impact of PD-L1 expression in patients with GC. The median follow-up period for censored patients was 3.7 years. During this time, 54 patients $(28.3 \%)$ experienced recurrence and 64 (33.5\%) died. Kaplan-Meier curves for OS and RFS are shown in Fig. 3. OS and RFS for patients with PD-L1 positivity by TPS were not significantly different than those for patients with PD-L1 negativity by TPS (Fig. 3a, b). In contrast, patients with PD-L1 positivity by CPS experienced significantly shorter OS $(P<0.01)$ and RFS $(P=0.01)$ times than patients with PD-L1 positivity by CPS (Fig. 3c, d).

Cox regression analysis was then performed for OS and RFS with regard to clinicopathological factors including age, sex, histological type, tumor markers, tumor (T) stage, node (N) stage, and PD-L1 expression by TPS and CPS (Tables 2, 3). Among these, high carcinoembryonic antigen (CEA) (HR 1.88, 95\% CI 1.08-3.17, $P=0.03$ ), high carbohydrate antigen 19-9 (CA19-9) (HR 1.88, 95\% CI $1.02-3.27, P=0.04$ ), and PD-L1 positivity by CPS (HR $2.29,95 \%$ CI $1.28-4.40, P<0.01$ ) had significantly worse OS in univariate analysis. Moreover, multivariate Cox regression analysis for OS demonstrated that PD-L1 positivity by CPS was a significant independent factor for poor prognosis (HR 2.27, 95\% CI 1.27-4.37, $P<0.01$ ). In addition, univariate analysis demonstrated male (HR 1.96, 95\% CI 1.17-3.47, $P=0.01$ ), high CA19-9 (HR 2.97, 95\% CI $1.72-4.92, P<0.01$ ), T3-T4 stage (HR 1.90, 95\% CI $1.13-3.36, P=0.01$ ), N1-N3 stage (HR $1.75,95 \%$ CI $1.11-2.77, P=0.02$ ), and PD-L1 positivity by CPS (HR $1.90,95 \%$ CI $1.13-3.36, P=0.01$ ) had worse RFS with statistical significance. Finally, multivariate Cox regression analysis demonstrated that high CA19-9 (HR 2.41, 95\% CI 1.36-4.09, $P<0.01$ ), T3-T4 stage (HR 1.70, 95\% CI $1.12-2.75, P<0.04)$ and PD-L1 positivity by CPS (HR 

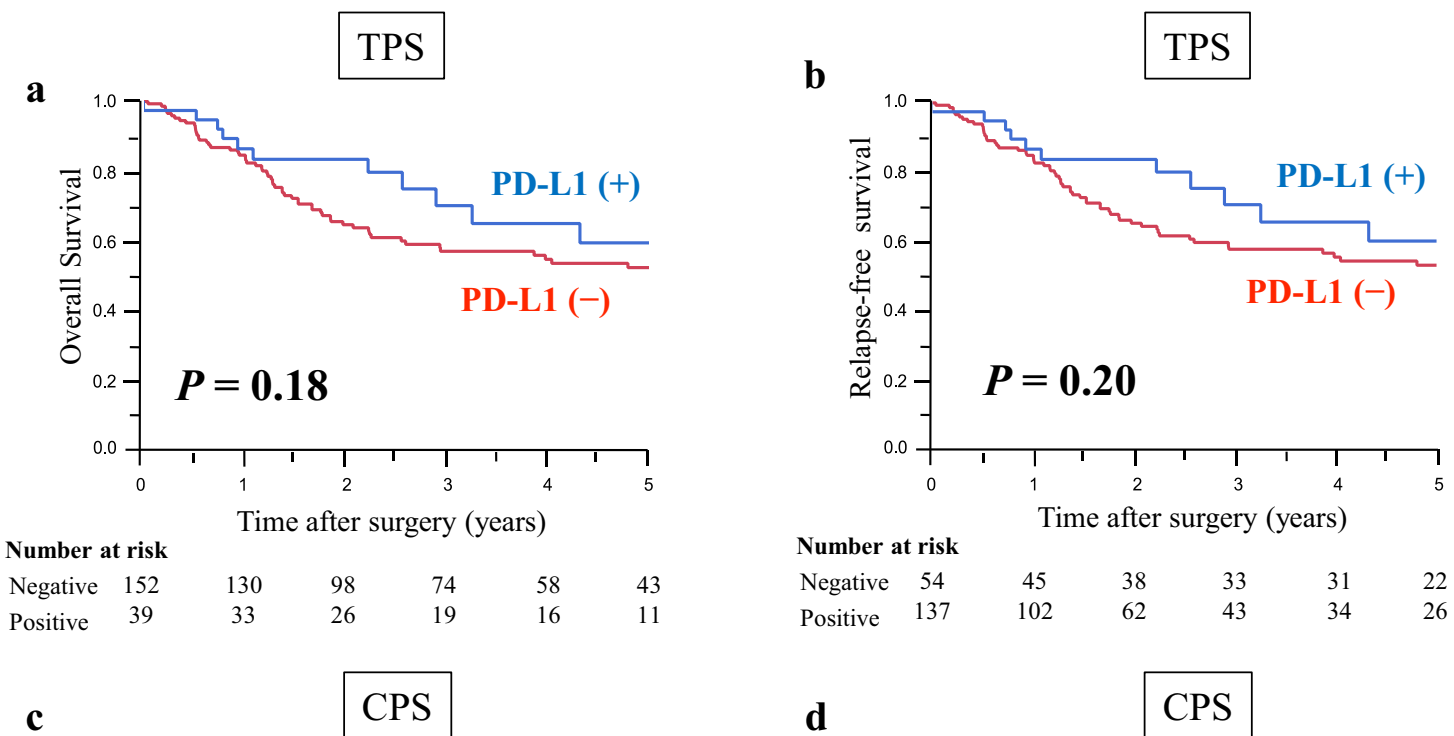

\begin{tabular}{lcccccc} 
Number at risk & \multicolumn{5}{l}{ (1) } \\
Negative & 54 & 45 & 38 & 33 & 31 & 22 \\
Positive & 137 & 102 & 62 & 43 & 34 & 26
\end{tabular}
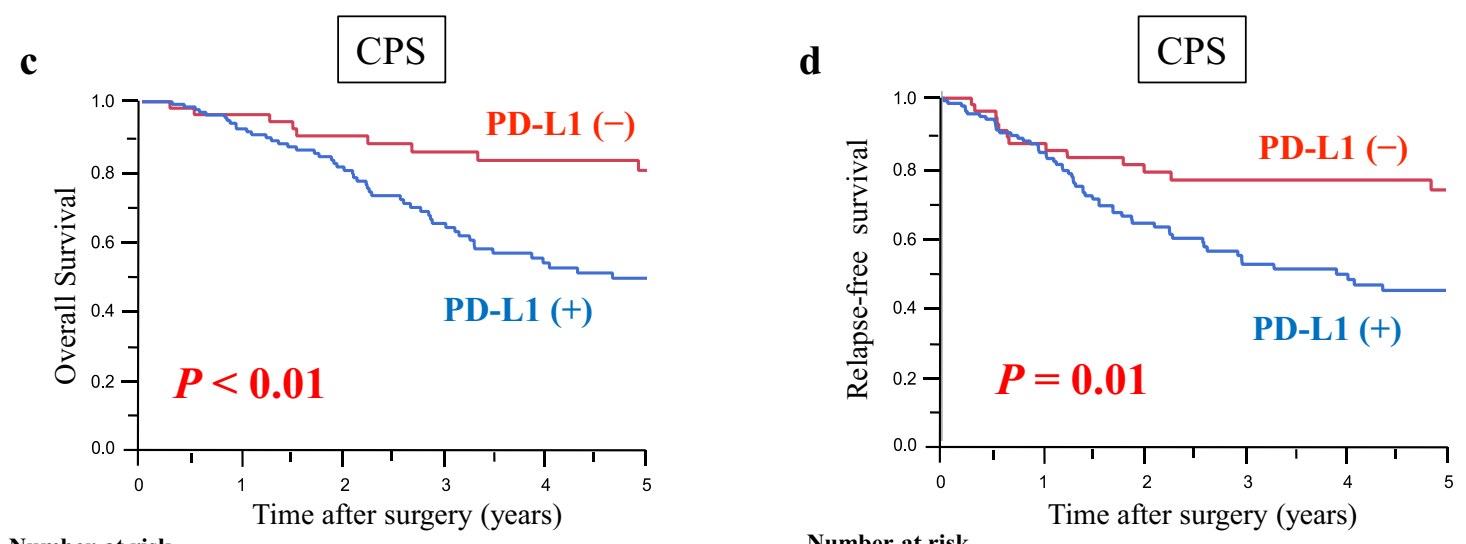

Number at risk

$\begin{array}{lllllll}\text { Negative } & 54 & 45 & 38 & 33 & 31 & 22\end{array}$

$\begin{array}{lcccccc}\text { Negative } & 54 & 50 & 43 & 37 & 34 & 25 \\ \text { Positive } & 137 & 113 & 81 & 56 & 40 & 29\end{array}$

Fig. 3 Kaplan-Meier curves for overall survival (OS) and relapsefree survival (RFS) according to PD-L1 expression. a OS for PDL1-positive and -negative groups by TPS. b RFS for PD-L1-positive

and -negative groups by TPS. c OS for PD-L1-positive and -negative groups by CPS. d RFS for PD-L1-positive and -negative groups by CPS

\section{Discussion}

$1.81,95 \%$ CI $1.07-3.22, P=0.03)$ were independent poor prognostic factor for RFS.

\section{Comprehensive analysis of PD-L1 expression by CPS and TILs for patient survival}

We then performed comprehensive analysis for OS and RFS in combination with PD-L1 expression by CPS and TILs. Kaplan-Meier curves for OS and RFS demonstrated that patients with PD-L1 negativity by CPS and TIL high status showed significantly better OS and RFS than those with PD-L1 negativity by CPS and TIL low status whereas patients with PD-L1 positivity by CPS and TIL low status showed worse OS and RFS than those with PD-L1 positivity by CPS and TIL high status ( $p<0.01$ and $p=0.03$, respectively; Fig. 4).

In this study, we revealed that patients with PD-L1 positivity by CPS experienced significantly shorter OS and RFS times than those with PD-L1 negativity by CPS. Moreover, multivariate Cox regression analysis for OS demonstrated that PD-L1 positivity by CPS was an independent factor for poor prognosis. Most previous reports on the prognostic impact of PD-L1 expression in GC focused on PD-L1 expression in tumor cells alone [5, 6]. Therefore, to our knowledge, this is the first study to demonstrate that PD-L1 expression by CPS is a more sensitive prognostic biomarker than PD-L1 expression by TPS in GC.

The relationship between PD-L1 expression in tumor cells and prognosis in GC is controversial. Some previous studies indicated that PD-L1 positivity was related to poor prognosis $[14,15]$, whereas other reports contradicted these findings $[16,17]$. Although a few meta-analyses reported 
Table 2 Univariate and multivariate analyses for overall survival

\begin{tabular}{|c|c|c|c|c|}
\hline & \multicolumn{2}{|l|}{ Univariate } & \multicolumn{2}{|l|}{ Multivariate } \\
\hline & HR (95\% CI) & $P$ value & HR (95\% CI) & $P$ value \\
\hline \multicolumn{5}{|l|}{ Age (years) } \\
\hline$\geq 65$ & $1.60(0.92-2.96)$ & 0.10 & & \\
\hline \multicolumn{5}{|l|}{ Sex } \\
\hline Male & $1.54(0.90-2.75)$ & 0.12 & & \\
\hline \multicolumn{5}{|l|}{ Histological type } \\
\hline Undifferentiated & $1.32(0.81-2.16)$ & 0.26 & & \\
\hline \multicolumn{5}{|l|}{ Tumor marker } \\
\hline CEA $>3.4$ & $1.88(1.08-3.17)$ & 0.03 & $1.64(0.92-2.85)$ & 0.09 \\
\hline CA19-9>37 & $1.88(1.02-3.27)$ & 0.04 & $1.62(0.86-2.89)$ & 0.14 \\
\hline \multicolumn{5}{|l|}{ PD-L1 expression } \\
\hline Positive (TPS) & $0.73(0.35-1.38)$ & 0.35 & & \\
\hline Positive (CPS) & $2.29(1.28-4.40)$ & $<0.01$ & $2.27(1.27-4.37)$ & $<0.01$ \\
\hline \multicolumn{5}{|l|}{ T stage } \\
\hline T3-T4 & $1.01(0.60-1.67)$ & 0.96 & & \\
\hline \multicolumn{5}{|l|}{$\mathrm{N}$ stage } \\
\hline N1-N3 & $1.49(0.91-2.44)$ & 0.11 & & \\
\hline
\end{tabular}

Statistically significant $P$ values are in bold $(P<0.05)$

$H R$ hazard ratio, $C I$ confidence interval

\begin{tabular}{|c|c|c|c|c|}
\hline & \multicolumn{2}{|l|}{ Univariate } & \multicolumn{2}{|l|}{ Multivariate } \\
\hline & HR (95\% CI) & $P$ value & HR $(95 \%$ CI $)$ & $P$ value \\
\hline \multicolumn{5}{|l|}{ Age (years) } \\
\hline$\geq 65$ & $1.53(0.91-2.70)$ & 0.11 & & \\
\hline \multicolumn{5}{|l|}{ Sex } \\
\hline Male & $1.96(1.17-3.47)$ & 0.01 & $1.72(0.96-3.08)$ & 0.05 \\
\hline \multicolumn{5}{|l|}{ Histological type } \\
\hline Undifferentiated & $1.04(0.67-1.64)$ & 0.86 & & \\
\hline \multicolumn{5}{|l|}{ Tumor marker } \\
\hline CEA $>3.4$ & $1.42(0.84-2.32)$ & 0.19 & & \\
\hline CA19-9> 37 & $2.97(1.72-4.92)$ & $<0.01$ & $2.41(1.36-4.09)$ & $<0.01$ \\
\hline \multicolumn{5}{|l|}{ PD-L1 expression } \\
\hline Positive (TPS) & $0.64(0.32-1.16)$ & 0.15 & & \\
\hline Positive (CPS) & $1.90(1.13-3.36)$ & 0.01 & $1.81(1.07-3.22)$ & 0.03 \\
\hline \multicolumn{5}{|l|}{ T stage } \\
\hline T3-T4 & $1.90(1.15-3.06)$ & 0.01 & $1.70(1.02-2.75)$ & 0.04 \\
\hline \multicolumn{5}{|l|}{ N stage } \\
\hline N1-N3 & $1.75(1.11-2.77)$ & 0.02 & $1.48(0.93-2.38)$ & 0.10 \\
\hline
\end{tabular}

Statistically significant $P$ values are in bold $(P<0.05)$

$H R$ hazard ratio, $C I$ confidence interval
Table 3 Univariate and multivariate analyses for relapse-free survival that PD-L1 positivity in tumor cells was a factor for worse prognosis, these studies reported several limitations including publication bias suggesting that studies refuting PD-L1 as a prognostic biomarker are less likely to be published $[5,6]$. Past studies obtained widely varying rates of positive PD-L1 expression, ranging from 14 to $69 \%$ [18]. The most likely reason for this discrepancy is the difference in assessment methods used to distinguish positive and negative PD-L1 expression. Because variations in frequency strongly affect whether PD-L1 expression in tumor cells could be a prognostic biomarker, standardized methods and definitions of PD-L1 positivity are needed.

Given that assessment methods of PD-L1 expression may influence the importance of PD-L1 expression as a 


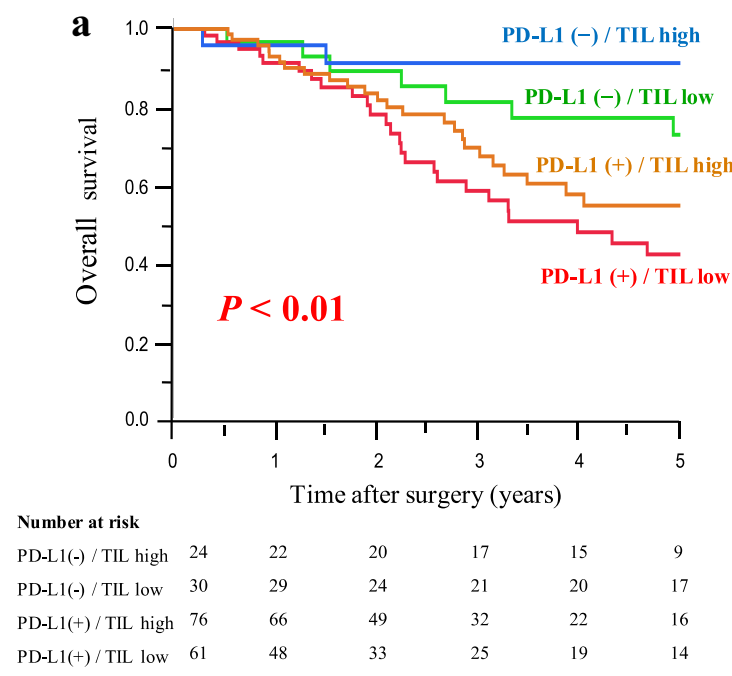

Fig. 4 Kaplan-Meier curves for overall survival (OS) and relapsefree survival (RFS) in combination with PD-L1 expression by CPS and TILs status. a OS for PD-L1-positive and -negative groups by

biomarker, several important clinical trials on the efficacy of PD-1 inhibitors in GC have adopted TPS with cut-off values of $1 \%$ as a measure of PD-L1 positivity [9]. However, this scoring system is still associated with the specific GC problem that it is difficult to histomorphologically distinguish poorly differentiated adenocarcinoma and macrophages expressing PD-L1, as shown in the present study (Fig. 1a, b). Therefore, we performed double IHC staining to clearly distinguish the two cell types, and achieved an accurate assessment of PD-L1 expression by TPS (Fig. 1c). Consequently, PD-L1 expression of tumor cells evaluated by TPS could not be a prognostic biomarker in this study. This result suggested that the assessment of PD-L1 expression in tumor cells alone cannot reflect the total immune tolerance state and may be inappropriate as a prognostic biomarker.

The impact of PD-L1 expression in immune cells is a key area of research interest because it induces immune tolerance [19]. To evaluate PD-L1 expression in immune cells, the mononuclear immune cell density score was developed [20], but this showed poor reproducibility in preliminary studies [11]. Therefore, the modified proportion score, CPS, was developed. In CPS, it is not necessary to distinguish PD-L1 expression between tumor cells and immune cells because both are taken into account. Moreover, CPS was reported to be a highly reproducible scoring system [11] that is becoming increasingly popular in assessment of the PD-L1 status as a predictive biomarker for PD-1 inhibitors in GC $[8,9]$.

In this study, we demonstrated the prognostic importance of PD-L1 expression by CPS, although clinicopathological factors were almost identical between PD-L1-positive and -negative patients. These results suggest that the total

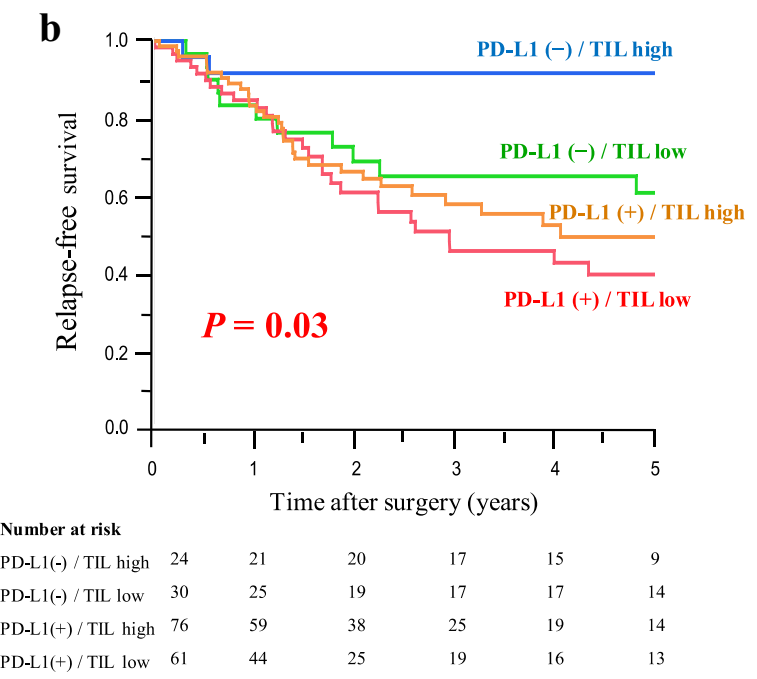

CPS in combination with TILs status. b RFS for PD-L1-positive and -negative groups by CPS in combination with TILs status

number of PD-L1-expressing cells, including tumor cells and immune cells, is a more sensitive biomarker than the number of PD-L1-expressing tumor cells in GC. Several studies using mouse model demonstrated that PD-L1 on both tumor cells and host immune cells contributed to immune suppression [21-23]. Based on these findings, we assume that the relative contributions of PD-L1 on these cells are context-dependent, CPS can evaluate all the PD-L1 expression in tumor microenvironment and thus can reflect not only the tumor malignant potential but also host immune status. Moreover, we also revealed that the combination analysis of PD-L1 expression by CPS and TILs status achieved a more sensitive prognostic stratification. Previous studies reported a classification of tumors into four groups based on PD-L1 expression and TIL status and the prognostic implication, which are consistent with our finding [24, 25]. We believe that these findings help us to improve risk-adapted therapeutic strategies and to stratify patients in future clinical trials for treatment with PD-1 inhibitors.

This study has several limitations. First, it was a retrospective study from a single institution. A multicenter study involving a large cohort should therefore be performed after the establishment of standard assessment methods for PD-L1 expression in GC to validate our findings. Second, several primary antibodies against PD-L1 are available but no consensus has been reached about which should be used for PD-L1 assessment in GC. Although some studies have compared cell staining using several PD-L1 antibodies [26], further work is needed to identify the optimal primary antibody against PD-L1 for use in GC. Third, we determined the cut-off value of 1 for PD-L1 positivity based on previous pivotal clinical 
trials about the efficacy of PD-1 inhibitors in GC [7, 8]. However, it was unclear whether this cut-off value could be applied to assess the prognostic importance of PD-L1 expression. The optimal cut-off value should be determined in a larger cohort, multicenter study.

In conclusion, we demonstrated that CPS is a more useful assessment method for PD-L1 expression than TPS as prognostic biomarkers. This result suggests that total PD-L1 expression in tumor cells and immune cells is associated with poor prognosis in GC rather than PD-L1 expression in tumor cells alone. Our study should be helpful in further discussion about whether PD-L1 expression could be used as a predictive biomarker in studies of PD-1 inhibitors in GC.

Acknowledgements We thank Sarah Williams, PhD, from Edanz Group (www.edanzediting.com) for editing a draft of this manuscript.

Funding This work was supported in part by the following grant and foundation: Japan Society for the Promotion of Science Grant-in-Aid for Scientific Research; Grant no. 16KK0184.

\section{Compliance with ethical standards}

Conflict of interest The authors declare that they have no conflict of interest.

Ethical standards All procedures followed were in accordance with the ethical standards of the responsible committee on human experimentation (institutional and national) and with the Helsinki Declaration of 1964 and later versions. Informed consent to be included in the study, or the equivalent, was obtained from all patients.

\section{References}

1. Bray F, Ferlay J, Soerjomataram I, Siegel RL, Torre LA, Jemal A. Global cancer statistics 2018: GLOBOCAN estimates of incidence and mortality worldwide for 36 cancers in 185 countries. CA Cancer J Clin. 2018;68:394-424.

2. Pardoll DM. The blockade of immune checkpoints in cancer immunotherapy. Nat Rev Cancer. 2012;12:252-64.

3. Zhou C, Tang J, Sun H, Zheng X, Li Z, Sun T, et al. PD-L1 expression as poor prognostic factor in patients with non-squamous non-small cell lung cancer. Oncotarget. 2017;8:58457-68.

4. Massi D, Brusa D, Merelli B, Falcone C, Xue G, Carobbio A, et al. The status of PD-L1 and tumor-infiltrating immune cells predict resistance and poor prognosis in BRAFi-treated melanoma patients harboring mutant BRAFV600. Ann Oncol. 2015;26:1980-7.

5. Zhang M, Dong Y, Liu H, Wang Y, Zhao S, Xuan Q, et al. The clinicopathological and prognostic significance of PD-L1 expression in gastric cancer: a meta-analysis of 10 studies with 1901 patients. Sci Rep. 2016;6:37933.

6. Gu L, Chen M, Guo D, Zhu H, Zhang W, Pan J, et al. PD-L1 and gastric cancer prognosis: a systematic review and meta-analysis. PLoS ONE. 2017;12:e0182692.

7. Kang YK, Boku N, Satoh T, Ryu MH, Chao Y, Kato K, et al. Nivolumab in patients with advanced gastric or gastro-oesophageal junction cancer refractory to, or intolerant of, at least two previous chemotherapy regimens (ONO-453812, ATTRACTION-2): a randomised, double-blind, placebocontrolled, phase 3 trial. Lancet. 2017;390:2461-71.

8. Fuchs CS, Doi T, Jang RW, Muro K, Satoh T, Machado M, et al. Safety and efficacy of pembrolizumab monotherapy in patients with previously treated advanced gastric and gastroesophageal junction cancer: phase 2 clinical KEYNOTE-059 trial. JAMA Oncol. 2018;4:e180013.

9. Shitara K, Ozguroglu M, Bang YJ, Di Bartolomeo M, Mandala $\mathrm{M}$, Ryu MH, et al. Pembrolizumab versus paclitaxel for previously treated, advanced gastric or gastro-oesophageal junction cancer (KEYNOTE-061): a randomised, open-label, controlled, phase 3 trial. Lancet. 2018;392:123-33.

10. Amin MB, Greene FL, Edge SB, Compton CC, Gershenwald JE, Brookland RK, et al. The Eighth Edition AJCC cancer staging manual: continuing to build a bridge from a population-based to a more "personalized" approach to cancer staging. CA Cancer J Clin. 2017;67:93-9.

11. Kulangara K, Zhang N, Corigliano E, Guerrero L, Waldroup S, Jaiswal D, et al. Clinical utility of the combined positive score for programmed death ligand-1 expression and the approval of pembrolizumab for treatment of gastric cancer. Arch Pathol Lab Med. 2018;143:330-7.

12. Kang BW, Seo AN, Yoon S, Bae HI, Jeon SW, Kwon OK, et al. Prognostic value of tumor-infiltrating lymphocytes in Epstein-Barr virus-associated gastric cancer. Ann Oncol. 2016;27:494-501.

13. Zhang D, He W, Wu C, Tan Y, He Y, Xu B, et al. Scoring system for tumor-infiltrating lymphocytes and its prognostic value for gastric cancer. Front Immunol. 2019;10:71.

14. Geng Y, Wang H, Lu C, Li Q, Xu B, Jiang J, et al. Expression of costimulatory molecules B7-H1, B7-H4 and Foxp3+ Tregs in gastric cancer and its clinical significance. Int $\mathrm{J}$ Clin Oncol. 2015;20:273-81.

15. Tamura T, Ohira M, Tanaka H, Muguruma K, Toyokawa T, Kubo $\mathrm{N}$, et al. Programmed death-1 ligand-1 (PDL1) expression is associated with the prognosis of patients with stage II/III gastric cancer. Anticancer Res. 2015;35:5369-76.

16. Boger C, Behrens HM, Mathiak M, Kruger S, Kalthoff H, Rocken C. PD-L1 is an independent prognostic predictor in gastric cancer of Western patients. Oncotarget. 2016;7:24269-83.

17. Kim JW, Nam KH, Ahn SH, Park DJ, Kim HH, Kim SH, et al. Prognostic implications of immunosuppressive protein expression in tumors as well as immune cell infiltration within the tumor microenvironment in gastric cancer. Gastric Cancer. 2016;19:42-52.

18. Harada K, Dong X, Estrella JS, Correa AM, Xu Y, Hofstetter WL, et al. Tumor-associated macrophage infiltration is highly associated with PD-L1 expression in gastric adenocarcinoma. Gastric Cancer. 2018;21:31-40.

19. Havel JJ, Chowell D, Chan TA. The evolving landscape of biomarkers for checkpoint inhibitor immunotherapy. Nat Rev Cancer. 2019;19:133-50.

20. Bellmunt J, Mullane SA, Werner L, Fay AP, Callea M, Leow $\mathrm{JJ}$, et al. Association of PD-L1 expression on tumor-infiltrating mononuclear cells and overall survival in patients with urothelial carcinoma. Ann Oncol. 2015;26:812-7.

21. Kleinovink JW, Marijt KA, Schoonderwoerd MJA, van Hall T, Ossendorp F, Fransen MF. PD-L1 expression on malignant cells is no prerequisite for checkpoint therapy. Oncoimmunology. 2017;6:e1294299.

22. Noguchi T, Ward JP, Gubin MM, Arthur CD, Lee SH, Hundal $\mathrm{J}$, et al. Temporally distinct PD-L1 expression by tumor and host cells contributes to immune escape. Cancer Immunol Res. 2017;5:106-17. 
23. Lau J, Cheung J, Navarro A, Lianoglou S, Haley B, Totpal K, et al. Tumour and host cell PD-L1 is required to mediate suppression of anti-tumour immunity in mice. Nat Commun. 2017;8:14572.

24. Teng MW, Ngiow SF, Ribas A, Smyth MJ. Classifying cancers based on T-cell infiltration and PD-L1. Cancer Res. 2015;75:2139-45.

25. Yagi T, Baba Y, Ishimoto T, Iwatsuki M, Miyamoto Y, Yoshida $\mathrm{N}$, et al. PD-L1 expression, tumor-infiltrating lymphocytes, and clinical outcome in patients with surgically resected esophageal cancer. Ann Surg. 2019;269:471-8.
26. Ma J, Li J, Qian M, Han W, Tian M, Li Z, et al. PD-L1 expression and the prognostic significance in gastric cancer: a retrospective comparison of three PD-L1 antibody clones (SP142, 28-8 and E1L3N). Diagn Pathol. 2018;13:91.

Publisher's Note Springer Nature remains neutral with regard to jurisdictional claims in published maps and institutional affiliations. 